\title{
Exposição crônica ao cloridrato de metformina e à glibenclamida causa alterações comportamentais, glicêmicas e de mortalidade em Hemigrammus caudovittatus e Danio rerio
}

\author{
[Chronic exposure to metformin chloridrate and glibenclamide causes behavioral, blood \\ glucose and mortality changes of Hemigrammus caudovittatus and Danio rerio] \\ E.Q. Souza ${ }^{1}$, T.Q.M. Bittencourt ${ }^{2}$, R.C.B. Ferreira ${ }^{1}$, E.G.S. Oliveira ${ }^{1}$, \\ N.P.C. Silva', S.C.B.L. Silva', M.R.S. Cadena ${ }^{3}$, P.G. Cadena ${ }^{3 *}$ \\ ${ }^{1}$ Progama de pós-graduação - Universidade Federal Rural de Pernambuco - Recife, PE \\ ${ }^{2}$ Aluno de graduação - Universidade Federal Rural de Pernambuco - Recife, PE \\ ${ }^{3}$ Universidade Federal Rural de Pernambuco - Recife, PE
}

\begin{abstract}
RESUMO
Hemigrammus caudovittatus e Danio rerio foram expostos aos hipoglicemiantes orais (HOs) cloridrato de metformina a $40 \mu \mathrm{g} / \mathrm{L}$ e $120 \mu \mathrm{g} / \mathrm{L}$ e glibenclamida a $0,13 \mu \mathrm{g} / \mathrm{L}$ e $0,39 \mu \mathrm{g} / \mathrm{L}$ durante 100 dias. Foram avaliados os efeitos tóxicos dos fármacos em relação ao peso, ao comportamento animal, à glicemia e à mortalidade. $H$. caudovittatus expostos à menor concentração dos fármacos apresentaram aumento significativo $(\mathrm{P}<0,05)$ no evento Respiração Aérea. Ainda, foi observado aumento no comportamento Descansar quando os animais foram expostos à glibenclamida a $0,39 \mu \mathrm{g} / \mathrm{L}$. Em D. rerio expostos ao cloridrato de metformina a $120 \mu \mathrm{g} / \mathrm{L}$, foi observado aumento $(\mathrm{P}<0,05)$ no comportamento Descansar. A glibenclamida provocou redução $(\mathrm{P}<0,05)$ na glicemia de $H$. caudovittatus. Ambos os fármacos causaram efeito letal na espécie $D$. rerio, contudo a glibenclamida foi mais tóxica, causando $100 \%$ de mortalidade em 30 dias de exposição. Os animais que vieram a óbito apresentaram congestão nos arcos branquiais e hemorragia. Os HOs foram desenvolvidos para apresentarem efeitos fisiológicos em mamíferos, entretanto efeitos tóxicos foram encontrados nas duas espécies de peixe estudadas. Isso levanta a preocupação sobre possíveis efeitos tóxicos de HOs e sobre quais métodos serão utilizados para a sua degradação no ambiente aquático.
\end{abstract}

Palavras-chave: peixe, comportamento animal, hipoglicemiante oral, ecotoxicologia

\begin{abstract}
Hemigrammus caudovittatus and Danio rerio were exposed to oral hypoglycemic drugs (HOs) metformin hydrochloride at $40 \mu \mathrm{g} / \mathrm{L}$ and $120 \mu \mathrm{g} / \mathrm{L}$ and to glibenclamide at $0.13 \mu \mathrm{g} / \mathrm{L}$ and $0.39 \mu \mathrm{g} / \mathrm{L}$ during 100 days. Toxic effects of the drugs were evaluated based on weight, animal behavior, blood glucose and mortality. $\mathrm{H}$. caudovittatus exposed to lowest concentration of the drugs showed significant increase $(P<0.05)$ in the Air breathing event. Furthermore, increase in Rest event was observed when animals were exposed to glibenclamide at $0.39 \mu \mathrm{g} / \mathrm{L}$. An increase $(P<0.05)$ in the frequency of Rest behavior in the $\mathrm{D}$. rerio exposed to metformin hydrochloride at $120 \mu \mathrm{g} / \mathrm{L}$ was observed. Glibenclamide caused decrease $(P<0.05)$ in the blood glucose of $\mathrm{H}$. caudovittatus. Both drugs caused lethal effect against $\mathrm{D}$. rerio. Nevertheless, glibenclamide was more toxic causing 100\% of mortality after 30 days of exposure. The animals that died showed congestion on the branchial arches and hemorrhage. The HOs were developed to have physiological effects in mammals. However, toxic effects were found in both species of fish studied. This raises concerns about possible toxic effects of HOs and what methods will be used for their degradation in the aquatic environment.
\end{abstract}

Keywords: fish, animal behavior, oral hypoglycemic, ecotoxicology

Recebido em 25 de junho de 2018

Aceito em 28 de dezembro de 2018

*Autor para correspondência (corresponding author)

E-mail: pabyton.cadena@ufrpe.br 


\section{INTRODUÇÃO}

Os gastos com o tratamento do diabetes no Brasil irão dobrar até 2030 segundo previsão de Bommer et al. (2018), sendo os hipoglicemiantes orais (HOs) a principal escolha para o tratamento de pacientes que não tiveram respostas a medidas não farmacológicas isoladas (Martínez-Viveros et al., 2018). Entre os HOs comercializados que estão presentes na lista de medicamentos essenciais da Organização Mundial da Saúde (2017), há o cloridrato de metformina, do grupo das biguanidas, que age aumentando a sensibilidade da insulina e reduzindo a taxa de gliconeogênese hepática (Pryor e Cabreiro, 2015), e a glibenclamida, do grupo das sulfonilureias de segunda geração, que estimula a secreção de insulina e reduz a produção hepática de glicose (Silva et al., 2009).

Devido ao grande uso dos HOs, ocorre a liberação desses compostos no meio ambiente, seja pela urina, seja pelas fezes, somada ao tratamento inadequado da água, o que resulta em concentrações de fármacos residuais, oriundos de hospitais e indústrias farmacêuticas ( $\mathrm{Li}$ e Lin, 2015). Com isso, concentrações relevantes de cloridrato de metformina e de glibenclamida foram encontradas em corpos d'água (Ternes et al., 2001; Niemuth et al., 2015). O cloridrato de metformina não é metabolizado, sendo liberado em sua forma inalterada no meio ambiente, por meio da urina humana (Niemuth et al., 2015). Também possui tempo de meia-vida no ambiente de cerca de 28 dias (Zielinski et al., 2002). Já em relação à glibenclamida, apenas $45 \%$ delas são absorvidas no trato gastrintestinal quando ingeridas por via oral (Obaidat e Ababneh, 2009), sendo o restante eliminado nas fezes. Um possível aumento nos gastos com o tratamento do diabetes no Brasil (Bommer et al., 2018) pode significar um aumento no uso desses fármacos, o que ocasionaria concentrações cada vez mais relevantes deles no meio ambiente. Isso gera preocupação sobre os efeitos tóxicos para os seres humanos e outros animais, incluindo os peixes, uma vez que a metformina pode atuar como um disruptor endócrino (Niemuth e Klaper, 2018). Substâncias que tenham ação de desregulação endócrina podem causar alterações comportamentais e reprodutivas, podendo levar a desequilíbrios populacionais em ambiente aquático (Santos et al., 2016).
Diante disso, testes toxicológicos utilizando peixes como modelo animal são de importância para o entendimento dos efeitos da exposição a produtos químicos, devido a sua semelhança fisiológica com modelos tradicionais, ao baixo custo de manutenção e à alta fecundidade (Planchart et al., 2016). Danio rerio (zebrafish) é um excelente e conhecido modelo animal para o estudo de doenças humanas, como diabetes (Connaughton et al., 2016) e ecotoxicidade (Planchart et al., 2016). Porém o Danio rerio não é encontrado nativamente na América do Sul, o que levanta a questão da importância do uso de espécies nativas de clima tropical para avaliar se a exposição a produtos químicos afeta de forma diferente essas espécies. Para não se limitar a uma única espécie de peixe, novos teleósteos comuns nas águas sul-americanas surgem para inovar a pesquisa em laboratório, como Hemigrammus caudovittatus (Tetra Buenos Aires), que, apesar de ser um peixe popular na aquicultura (Kucharczyk et al., 2008), foi pouco utilizado em testes toxicológicos. Diante do exposto, o presente trabalho teve como objetivo avaliar os efeitos tóxicos causados pela exposição em concentrações encontradas no ambiente dos hipoglicemiantes orais (HOs) cloridrato de metformina e glibenclamida no comportamento e na fisiologia de Hemigrammus caudovittatus, uma espécie endêmica na América do Sul, e Danio rerio, uma espécie comum em testes toxicológicos.

\section{MATERIAL E MÉTODOS}

Os experimentos foram realizados no Laboratório de Ecofisiologia e Comportamento Animal - Leca da Universidade Federal Rural de Pernambuco, sendo previamente aprovados pela Comissão de Ética no Uso de Animais (CeuaUFRPE), licença 042/2017. Indivíduos das espécies Hemigrammus caudovittatus $(\mathrm{n}=60)$ e Danio rerio $(\mathrm{n}=60)$, adultos hígidos, foram adquiridos em criadouros comerciais e com proporção igual de machos e fêmeas. Posteriormente, foram aclimatados por 30 dias, para garantir a ausência de patógenos ou doenças, em aquários de 60 litros, oxigenado com canister JEBO $835(11 \mathrm{mg} / \mathrm{L}$ de oxigênio dissolvido), com fotoperíodo 14/10h e temperatura ambiente de $27 \pm 1^{\circ} \mathrm{C}$. A água foi tratada com adição de tiossulfato de sódio para a redução do cloro e de hidróxido de sódio para a

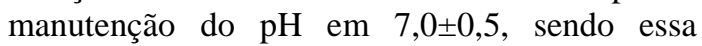


condição mantida durante todos os experimentos. Os animais foram alimentados ad libtum com ração comercial extrusada $(45 \%$ de proteína bruta), uma vez ao dia, de acordo com a metodologia de Roubach et al. (2002), que estabelece a quantidade de $2 \%$ a $5 \%(\mathrm{p} / \mathrm{g})$ de ração para animais adultos.

Posteriormente, $H$. caudovitattus e D. rerio foram divididos em três grupos $(\mathrm{n}=10$ por grupo com replicata autêntica), em aquários de oito 1itros, totalizando 120 animais. Os animais foram submetidos a um teste de toxicidade crônica por exposição química aos fármacos cloridrato de metformina a $40 \mu \mathrm{g} / \mathrm{L}$ (Niemuth el $a l ., 2015)$ e glibenclamida a $0,13 \mu \mathrm{g} / \mathrm{L}$ (Ternes et al., 2001) por 50 dias e grupo controle para cada espécie. Essas concentrações foram encontradas em águas residuais nos Estados Unidos e em rios da Alemanha. Após isso, houve um aumento da concentração para $120 \mu \mathrm{g} / \mathrm{L}$ e $0,39 \mu \mathrm{g} / \mathrm{L}$ de cloridrato de metformina e de glibenclamida, respectivamente, nos mesmos grupos, ocorrendo exposição por mais 50 dias. As concentrações foram triplicadas de forma arbitrária, levando em consideração um possível cenário de aumento de incidência de diabetes (Bommer et al., 2018) e consequente maior liberação desses fármacos no ambiente. As concentrações dos fármacos foram renovadas a cada semana, com a troca completa da água dos aquários, e os resíduos gerados durante o experimento passaram por tratamento por processo oxidativo avançado (POA) em reator utilizando foto-oxidação $\mathrm{UV}$ e $\mathrm{H}_{2} \mathrm{O}_{2}$ antes do descarte. Os parâmetros biológicos avaliados foram peso inicial e peso final, comportamento animal, glicemia e mortalidade.

Para mensurar o peso dos animais estudados, os pesos médios inicial e final de $H$. caudvitattus e $D$. rerio foram avaliados segundo metodologia descrita por Soares et al. (2016). Para a avaliação comportamental, foi utilizado um etograma para D. rerio, descrito previamente pelo presente grupo de pesquisa em Bittencourt et al. (2018) e modificado para o uso em ambas as espécies. Em H. caudovittatus foram adicionados comportamentos na categoria Locomoção: Natação Circular - NC (Natação em direção circular) e Nadar no Fundo - NF (Nadar próximo ao substrato); na categoria Social: Agregar - AG (Agregar-se aos demais peixes), Atacar - AT (Ataque com a boca no corpo do oponente) e
Afastar - AF (Afastar-se dos demais peixes); e na categoria Resposta ao Estresse: Emergir e Submergir - ES (Movimento rápido e repetitivo da superfície ao substrato). Ao etograma de Danio rerio foram adicionados os mesmos comportamentos, com exceção de Afastar e Emergir e Submergir, não observados nesses animais. A coleta dos dados comportamentais foi realizada pelo método de varredura instantânea (Soares et al., 2016; Altmann, 1974) duas vezes por semana, no período da tarde (14h), em uma distância fixa dos aquários $(1,5 \mathrm{~m})$ para não provocar estresse nos animais. Cada sessão teve duração de 30 minutos, alternando um minuto de observação e um minuto de intervalo para cada aquário. Nos dias de avaliação, o alimento permaneceu durante toda a análise para se verificarem comportamentos relacionados à alimentação, e posteriormente foi retirado.

Ao final de 100 dias de exposição, foi avaliada a glicemia dos animais. Para isso, os indivíduos foram submetidos a 24 horas de jejum (Connaughton et al., 2016) e anestesiados com eugenol 60mg/L (Grush et al., 2004). Com auxílio de seringa $(1 \mathrm{~mL})$, tiveram o sangue coletado ao longo do eixo do corpo e posterior ao ânus na região da aorta caudal (Zang et al., 2013). Para aferir a glicemia, utilizou-se um glicosímetro portátil (Gleeson et al., 2007) da marca G-Tech Free Lite, SD Biosensor inc. (Coreia do Sul). A mortalidade dos animais foi verificada diariamente, e os que foram a óbito tiveram a região opercular dissecada e analisada macroscopicamente em estereomicroscópio, para a averiguação da causa mortis. As análises estatísticas foram realizadas utilizando-se o Origin Pro Academic 2015 (Origin Lab. Northampton, MA USA). Quando o resultado foi significativo, as médias foram comparadas pelo teste de Tukey $(\mathrm{P}<0,05)$. Os dados foram apresentados na forma de média \pm desvio-padrão (DP).

\section{RESULTADOS}

Os resultados de pesos médios inicial e final de Hemigrammus caudovittatus e Danio rerio são apresentados na Tab. 1. Observa-se que não houve diferença significativa $(\mathrm{P}>0,05)$ no peso final dos animais submetidos à exposição química ao cloridrato de metformina e à glibenclamida. 
Exposição crônica ao...

Tabela 1. Efeito da exposição ao cloridrato de metformina e à glibenclamida nos pesos inicial e final (após 100 dias de exposição química) de Hemigrammus caudovittatus e Danio rerio

\begin{tabular}{lcccc} 
& & Controle & $\begin{array}{c}\text { Cloridrato de } \\
\text { metformina }\end{array}$ & Glibenclamida \\
\hline \multirow{2}{*}{ H. caudovitattus } & Peso inicial (g) & $3,39 \pm 0,79$ & $3,08 \pm 0,50$ & $3,12 \pm 0,69$ \\
& Peso final (g) & $3,75 \pm 0,83$ & $3,56 \pm 0,95$ & $3,66 \pm 0,89$ \\
D. rerio & Peso inicial (g) & $0,43 \pm 0,07$ & $0,46 \pm 0,09$ & $0,53 \pm 0,11$ \\
& Peso final (g) & $0,43 \pm 0,10$ & $0,41 \pm 0,17$ & $\mathrm{ND}^{\#}$ \\
\hline
\end{tabular}

$\mathrm{ND}^{\#}$ Os animais vieram a óbito antes do fim do experimento.

Os resultados da análise comportamental de Hemigrammus caudovittatus são apresentados na Tab. 2. Com relação aos animais expostos ao cloridrato de metformina na concentração de $40 \mu \mathrm{g} / \mathrm{L}$, houve um aumento $(\mathrm{P}<0,05)$ na exibição do comportamento Nadar Rápido. Na categoria Resposta ao Estresse, também houve um aumento $(\mathrm{P}<0,05)$ na exibição do comportamento Respiração Aérea. Quando a concentração foi aumentada para $120 \mu \mathrm{g} / \mathrm{L}$, observou-se um aumento $(\mathrm{P}<0,05)$ na exibição do comportamento Nadar Lento e uma redução em comportamentos da categoria Social (Agregar, Perseguir e Fugir). No entanto, o comportamento Respiração Aérea não diferiu do grupo controle, porém houve aumento $(\mathrm{P}<0,05)$ no comportamento Emergir e Submergir.
Com relação à glibenclamida, na concentração $0,13 \mu \mathrm{g} / \mathrm{L}, H$. caudovittatus apresentaram maior frequência $(\mathrm{P}<0,05)$ de exibição do comportamento Nadar Rápido, um comportamento ativo, e na categoria Resposta ao Estresse, houve um aumento $(\mathrm{P}<0,05)$ do comportamento Respiração Aérea. Entretanto, quando a concentração foi triplicada para $0,39 \mu \mathrm{g} / \mathrm{L}$, ocorreu um aumento $(\mathrm{P}<0,05)$ no comportamento Descansar, um comportamento inativo (Tab. 2). Na categoria Social, também houve uma redução $(\mathrm{P}<0,05)$ no comportamento Agregar e Afastar, e o comportamento Respiração Aérea não diferiu do grupo controle.

Tabela 2. Eventos comportamentais de Hemigrammus caudovittatus expostos ao cloridrato de metformina e à glibenclamida em diferentes concentrações. Legenda: C - controle (0-100 dias); M - cloridrato de

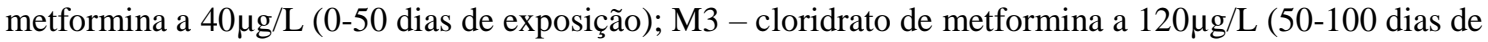
exposição); G - glibenclamida a $0,13 \mu \mathrm{g} / \mathrm{L}$ (0-50 dias de exposição); G3 - glibenclamida a $0,39 \mu \mathrm{g} / \mathrm{L}(50-$ 100 dias de exposição)

\begin{tabular}{cccccc}
\hline \multicolumn{5}{c}{ Hemigrammus caudovittatus } \\
\hline \multirow{2}{*}{ Comportamentos } & $\mathrm{C}$ & $\mathrm{M}$ & $\mathrm{M} 3$ & $\mathrm{G}$ & $\mathrm{G} 3$ \\
NL & $32,2 \pm 9,7$ & $33,1 \pm 7,8$ & $55,8 \pm 17,1^{*}$ & $28,3 \pm 14,7$ & $39,3 \pm 20,5$ \\
NR & $2,3 \pm 2,2$ & $11,9 \pm 9,5^{*}$ & $1,2 \pm 1,9$ & $8,8 \pm 4,1^{*}$ & $1,7 \pm 1,2$ \\
NC & $0,2 \pm 0,2$ & $0,1 \pm 0,1$ & $0,1 \pm 0,1$ & $0,1 \pm 0,2$ & $0,3 \pm 0,6$ \\
NF & $0,6 \pm 0,9$ & 0,0 & $0,0 \pm 0,1$ & $0,2 \pm 0,3$ & $0,1 \pm 0,1$ \\
FL & $22,7 \pm 10,5$ & $27,8 \pm 17,2$ & $23,7 \pm 16,5$ & $16,2 \pm 10,1$ & $17,6 \pm 9,2$ \\
DE & $7,6 \pm 5,6$ & $3,0 \pm 2,9$ & $9,7 \pm 6,1$ & $15,3 \pm 19,6$ & $31,1 \pm 19,7^{*}$ \\
FO & $0,1 \pm 0,1$ & $0,0 \pm 0,1$ & $0,1 \pm 0,1$ & $0,1 \pm 0,1$ & $0,0 \pm 0,1$ \\
CA & $1,6 \pm 1,4$ & $1,0 \pm 0,7$ & $3,1 \pm 2,7$ & $0,8 \pm 0,6$ & $0,6 \pm 0,5$ \\
AG & $19,1 \pm 12,1$ & $12,8 \pm 7,8$ & $2,3 \pm 3,5^{*}$ & $16,4 \pm 9,3$ & $1,4 \pm 2,3^{*}$ \\
PE & $5,1 \pm 2,6$ & $3,6 \pm 2,1$ & $1,7 \pm 1,3^{*}$ & $4,9 \pm 2,3$ & $3,3 \pm 2,8$ \\
FU & $5,6 \pm 2,9$ & $4,3 \pm 2,4$ & $1,8 \pm 1,4 *$ & $5,3 \pm 2,3$ & $3,8 \pm 3,0$ \\
AT & $0,5 \pm 0,6$ & $0,7 \pm 0,7$ & $0,1 \pm 0,1$ & $0,4 \pm 0,3$ & $0,4 \pm 0,4$ \\
AF & $1,9 \pm 1,0$ & $1,4 \pm 0,9$ & $0,9 \pm 1,1$ & $1,2 \pm 0,6$ & $0,2 \pm 0,3^{*}$ \\
ES & 0,0 & $1,4 \pm 3,3$ & $3,4 \pm 3,9^{*}$ & $0,0 \pm 0,0$ & 0,0 \\
RA & $0,1 \pm 0,1$ & $0,6 \pm 0,6^{*}$ & $0,1 \pm 0,2$ & $0,8 \pm 0,9^{*}$ & $0,2 \pm 0,3$ \\
\hline
\end{tabular}

Para os comportamentos exibidos na categoria Locomoção: NL - Nadar Lento; NR - Nadar Rápido; NC - Natação Circular; NF - Nadar no Fundo; FL - Flutuar; DE - Descansar. Na categoria Alimentação: FO - Forragear; CA Capturar. Na categoria Social: AG - Agregar; PE - Perseguir; FU - Fugir; AT - Atacar; AF - Afastar. Na categoria Resposta ao Estresse: ES - Emergir e Submergir; RA - Respiração Aérea. *Médias com diferença significativa $(\mathrm{P}<0,05)$ pelo teste de Tukey. 
Com relação à análise comportamental da espécie D. rerio (Tab. 3) exposta ao cloridrato de metformina, a exibição do comportamento Nadar Lento não diferiu $(\mathrm{P}>0,05)$ do grupo controle quando utilizada na concentração ambiental $(40 \mu \mathrm{g} / \mathrm{L})$, contudo, quando a concentração foi triplicada $(120 \mu \mathrm{g} / \mathrm{L})$, foi observada uma redução $(\mathrm{P}<0,05)$ nesse comportamento (Tab. 3). Nessa mesma concentração $(120 \mu \mathrm{g} / \mathrm{L})$, houve um aumento $(\mathrm{P}<0,05)$ da exibição do comportamento Descansar e de comportamentos agonísticos (Perseguir e Fugir) (Tab. 2). Adicionalmente, $D$. rerio expostos ao cloridrato de metformina a $40 \mu \mathrm{g} / \mathrm{L}$ e $120 \mu \mathrm{g} / \mathrm{L}$ e à glibenclamida a $0,13 \mu \mathrm{g} / \mathrm{L}$ apresentaram uma redução $(\mathrm{P}<0,05)$ na frequência do comportamento Capturar (Tab. 3).

Tabela 3. Eventos comportamentais de Danio rerio expostos ao cloridrato de metformina e à glibenclamida em diferentes concentrações. Legenda: C - controle (0-100 dias); $\mathrm{M}$ - cloridrato de

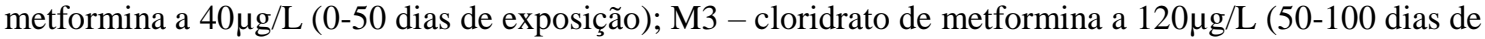
exposição); G - glibenclamida a $0,13 \mu \mathrm{g} / \mathrm{L}$ (0-50 dias de exposição); G3 - glibenclamida a 0,39 $\mu \mathrm{g} / \mathrm{L}$ (50100 dias de exposição)

\begin{tabular}{|c|c|c|c|c|c|}
\hline $\begin{array}{c}\text { Comportamento } \\
\mathrm{s}\end{array}$ & $\mathrm{C}$ & $\begin{array}{c}\mathrm{M} \\
(40 \mu \mathrm{g} / \mathrm{L})\end{array}$ & $\begin{array}{c}\text { Danio rerio } \\
\text { M3 } \\
(120 \mu \mathrm{g} / \mathrm{L})\end{array}$ & $\begin{array}{c}\mathrm{G} \\
(0,13 \mu \mathrm{g} / \mathrm{L})\end{array}$ & $\begin{array}{c}\mathrm{G} 3 \\
(0,39 \mu \mathrm{g} / \mathrm{L})\end{array}$ \\
\hline NL & $78,9 \pm 5,3$ & $70,8 \pm 5,6$ & $43,2 \pm 16,2 *$ & $71,4 \pm 17,1$ & $\mathrm{ND}^{\#}$ \\
\hline NR & $3,7 \pm 4,9$ & $3,6 \pm 1,5$ & $1,0 \pm 1,1$ & $5,5 \pm 4,3$ & $\mathrm{ND}^{\#}$ \\
\hline $\mathrm{NC}$ & 0,0 & $0,1 \pm 0,2$ & $0,2 \pm 0,4$ & 0,0 & $\mathrm{ND}^{\#}$ \\
\hline $\mathrm{NF}$ & 0,0 & 0,0 & 0,0 & 0,0 & $\mathrm{ND}^{\#}$ \\
\hline FL & $2,1 \pm 2,3$ & $2,4 \pm 2,9$ & $3,9 \pm 2,7$ & $4,9 \pm 7,3$ & $\mathrm{ND}^{\#}$ \\
\hline $\mathrm{DE}$ & $0,3 \pm 0,4$ & $0,7 \pm 0,9$ & $11,8 \pm 9,0 *$ & $4,7 \pm 7,8$ & $\mathrm{ND}^{\#}$ \\
\hline FO & $6,5 \pm 3,7$ & $12,2 \pm 5,9$ & $9,1 \pm 6,8$ & $6,9 \pm 8,8$ & $\mathrm{ND}^{\#}$ \\
\hline $\mathrm{CA}$ & $3,0 \pm 1,3$ & $0,9 \pm 0,8^{*}$ & $0,0^{*}$ & $0,2 \pm 0,3^{*}$ & $\mathrm{ND}^{\#}$ \\
\hline $\mathrm{AG}$ & $0,2 \pm 0,4$ & $0,3 \pm 0,6$ & 0,0 & $2,2 \pm 3,8$ & $\mathrm{ND}^{\#}$ \\
\hline PE & $2,5 \pm 1,4$ & $2,7 \pm 2,5$ & $14,4 \pm 9,4^{*}$ & $0,5 \pm 0,9 *$ & $\mathrm{ND}^{\#}$ \\
\hline FU & $2,5 \pm 1,4$ & $2,7 \pm 2,5$ & $14,6 \pm 8,7 *$ & $0,7 \pm 1,2$ & $\mathrm{ND}^{\#}$ \\
\hline $\mathrm{AT}$ & $0,2 \pm 0,2$ & 0,0 & $0,4 \pm 0,5$ & $0,2 \pm 0,3$ & $\mathrm{ND}^{\#}$ \\
\hline RA & $0,2 \pm 0,6$ & $3,7 \pm 6,6$ & $1,3 \pm 1,1$ & $2,9 \pm 5,0$ & $\mathrm{ND}^{\#}$ \\
\hline
\end{tabular}

Para os comportamentos exibidos na categoria Locomoção: NL - Nadar Lento; NR - Nadar Rápido; NC - Natação Circular; NF - Nadar no Fundo; FL - Flutuar; DE - Descansar. Na categoria Alimentação: FO - Forragear; CA Capturar. Na categoria Social: AG - Agregar; PE - Perseguir; FU - Fugir; AT - Atacar. Na categoria Resposta ao Estresse: RA - Respiração Aérea. *Médias com diferença significativa $(\mathrm{P}<0,05)$ pelo teste de Tukey. ND ${ }^{\#}$ Os animais vieram a óbito antes do fim do experimento.

Os resultados da aferição da glicemia nos animais após os 100 dias de exposição química são apresentados na Tab. 4. Com relação à avaliação da glicemia de $H$. caudovittatus, foi observada uma redução significativa $(\mathrm{P}<0,05)$ nos animais expostos à glibenclamida. Já em relação ao $D$. rerio, os animais vieram a óbito antes do fim do experimento, não sendo possível a aferição da glicemia em 100 dias de experimento.

Tabela 4. Efeitos da exposição química por 100 dias de cloridrato de metformina e de glibenclamida na glicemia (mg/dL) de Hemigramus caudovitattus e Danio rerio em teste de toxicidade crônica

\begin{tabular}{cccc} 
& Controle & Cloridrato de metformina & Glibenclamida \\
\hline H. caudovittatus & $94,2 \pm 29,5$ & $89,7 \pm 19,5$ & $68,8 \pm 19,9^{*}$ \\
D. rerio & $50,8 \pm 15,8$ & $\mathrm{ND}^{\#}$ & $\mathrm{ND}^{\#}$
\end{tabular}

*Médias com diferença significativa $(\mathrm{P}<0,05)$ pelo teste de Tukey. ND ${ }^{\#}$ Os animais vieram a óbito antes do fim do experimento.

Com relação à mortalidade de $H$. caudovittatus, foi observado resultado inferior a $10 \%$ em todos os grupos experimentais, indicando baixa letalidade dos fármacos nessa espécie. Já na espécie $D$. rerio, nos animais expostos ao cloridrato de metformina a $40 \mu \mathrm{g} / \mathrm{L}$, foi observada mortalidade de $20 \%$ dos animais a partir de 28 dias, e, ao aumentar a concentração para 
$120 \mu \mathrm{g} / \mathrm{L}$, foi observada mortalidade em $75 \%$ dos animais no final da exposição (100 dias). Ainda, em $D$. rerio, a partir de 24 dias, observou-se mortalidade no grupo exposto à glibenclamida a $0,13 \mu \mathrm{g} / \mathrm{L}$ com $100 \%$ de mortalidade em 30 dias de exposição química. Nos animais (D. rerio) expostos ao cloridrato de metformina, após a dissecação, foi possível observar congestão nos arcos branquiais, sendo em maior grau nos animais que foram expostos à maior concentração $(120 \mu \mathrm{g} / \mathrm{L})$. Nos animais expostos à glibenclamida a $0,13 \mu \mathrm{g} / \mathrm{L}$, foi possível observar, além da congestão nos arcos branquiais, hemorragia na região opercular, segmento medial e próximo da linha lateral (Figura 1).

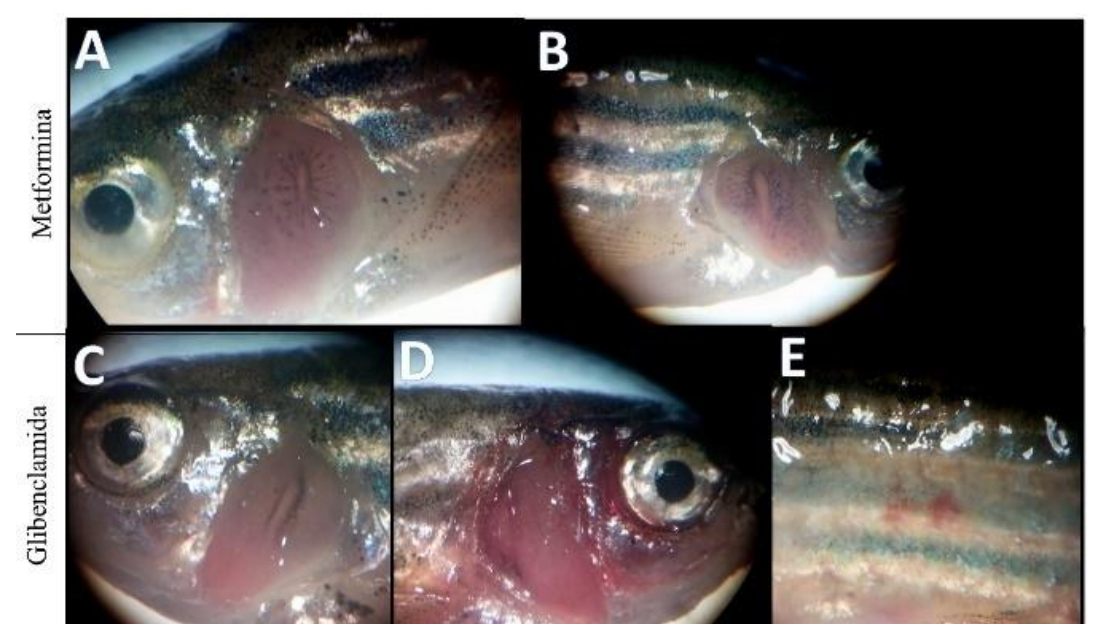

Figura 1. Danio rerio expostos ao cloridrato de metformina a $40 \mu \mathrm{g} / \mathrm{L}$ (A) e a $120 \mu \mathrm{g} / \mathrm{L}$ (B) e glibenclamida a $0,13 \mu \mathrm{g} / \mathrm{L}(\mathrm{C}, \mathrm{D}$ e E). A, B e C também representam congestão nos arcos branquiais. D e E também representam hemorragia na região opercular e segmento medial, respectivamente.

\section{DISCUSSÃO}

Os resultados obtidos indicaram que a exposição química ao cloridrato de metformina e à glibenclamida provocou efeitos tóxicos em $H$. caudovittatus e D. rerio em relação ao comportamento animal, à glicemia e à mortalidade. Entretanto, em relação ao ganho de peso dos animais, não foram observadas diferenças significativas $(\mathrm{P}>0,05)$. Niemuth el al. (2015) observaram uma redução no ganho de peso em machos de Pimephales promelas jovens expostos ao cloridrato de metformina $(40 \mu \mathrm{g} / \mathrm{L})$ por 320 dias, diferindo do presente estudo. Isso provavelmente ocorreu devido ao fato de que foram utilizados $H$. caudovittatus $e$ D. rerio adultos e em um tempo de exposição menor (100 dias).

Uma vez que o comportamento serve de ligação entre os processos físiológicos e ecológicos, pode ser ideal para estudar os efeitos de poluentes ambientais em baixas concentrações, utilizando peixes como modelo animal, já que comportamentos são facilmente observados e quantificados nesses animais (Scott e Sloman, 2004; MacLaren et al., 2018). Devido ao fato de o cloridrato de metformina ser considerado um disruptor endócrino que interfere com a expressão de genes relacionados ao metabolismo de hormônios esteroides, como o 17 $\beta$-estradiol, em peixes (Niemuth e Klaper, 2018), já eram esperadas alterações em comportamentos sociais nos resultados do presente estudo, como observado nas Tab. 2 e 3. Ainda, MacLaren et al. (2018) também observaram alterações em comportamentos sociais em Betta splendens expostos a concentrações ambientais de cloridrato de metformina $(40 \mu \mathrm{g} / \mathrm{L})$. Adicionalmente, Santos et al. (2016) relataram aumento de letargia (comportamentos inativos) em Betta splendens expostos ao $17 \beta$-estradiol e a outros esteroides, o que poderia justificar, no presente estudo, o aumento da frequência de exibição do comportamento Nadar Lento em ambas as espécies e do comportamento Descansar em D. rerio (Tab. 2 e 3). Também foram observados comportamentos associados ao estresse, como Respiração Aérea e Emergir e Submergir, em H. caudovittatus expostos a 40 e 
a $120 \mu \mathrm{g} / \mathrm{L}$. Sabe-se que o cloridrato de metformina pode alterar o metabolismo mitocondrial em peixes (Niemuth e Klaper, 2018), consequentemente o metabolismo aeróbico, o que poderia justificar a interferência nesses comportamentos.

Em relação à aferição de glicemia, não foram observadas alterações em $H$. caudovittatus expostos ao cloridrato de metformina (Tab. 4). A metformina não estimula a secreção de insulina, não tendo, por isso, ação hipoglicemiante (Metformina, 2015) (Pryor e Cabreiro, 2015) em indivíduos hígidos, o que poderia justificar o observado no presente estudo. O cloridrato de metformina pode alterar o metabolismo mitocondrial em peixes (Niemuth e Klaper, 2018), reduzindo o metabolismo aeróbico e tendo relação com a mortalidade. Isso poderia justificar a mortalidade dependente da concentração e congestão nos arcos branquiais encontrada durante a necropsia de $D$. rerio (Fig. 1). Ainda, é possível inferir que $H$. caudovittatus foi mais resistente à exposição química a esse poluente do que $D$. rerio, já que foram apenas observadas alterações comportamentais, incluindo comportamentos associados ao estresse (Respiração Aérea). D. rerio, além das alterações comportamentais, também exibiu alta mortalidade $(75 \%)$.

A exposição química à glibenclamida também provocou alterações comportamentais em ambas as espécies. Em $H$. caudovittatus foi observada, na concentração ambiental $(0,13 \mu \mathrm{g} / \mathrm{L})$, uma relação entre $\mathrm{o}$ aumento da frequência de exibição do comportamento Nadar Rápido e Respiração Aérea (Tab. 2). Glibenclamida aumentou a deposição de glicogênio no músculo esquelético do peixe Puntius conchonius (Khanna e Singh, 1983), o que poderia levar a um aumento da atividade locomotora e à maior procura por oxigênio. Com o aumento da concentração $(0,39 \mu \mathrm{g} / \mathrm{L})$, houve um efeito inverso, em que se observou um aumento acentuado do comportamento Descansar (Tab. 2), um comportamento inativo. Martínez-Viveros et al. (2018) observaram um aumento da oxidação proteica, dependente da concentração de glibenclamida na água, no músculo de Cyprinus carpio. Esse efeito tóxico pode reduzir a atividade muscular, o que poderia justificar o aumento do comportamento inativo encontrado no presente estudo. A glibenclamida reduziu a glicemia de $H$. caudovittatus (Tab. 4), assim como também observado por Martínez-Viveros et al. (2018) em Cyprinus carpio. As sulfonilureias, das quais a glibenclamida faz parte, são substâncias secretagogas da insulina que alteram o equilíbrio de secreção da insulina endógena pela célula betapancreática, aumentando a sua secreção (Elo et al., 2007). Os mesmos autores observaram esse efeito hipoglicemiante em glipzida, uma sulfonilureia com o mesmo mecanismo de ação da glibenclamida, por via endovenosa, em adultos de $D$. rerio. No presente estudo, observou-se o efeito hipoglicemiante em $H$. caudovittatus, com a glibenclamida presente na água, em concentração ambientalmente relevante.

D. rerio se mostrou um organismo mais sensível à exposição química à glibenclamida do que $H$. caudovittatus. A concentração ambiental $(0,13 \mu \mathrm{g} / \mathrm{L})$ provocou alterações em comportamentos sociais de D. rerio (Tab. 3), entretanto a literatura científica não descreve ações de disruptor endócrino para a glibenclamida, portanto esse efeito tóxico pode ter relação com algum mecanismo ainda não conhecido. Assim, estudos posteriores são necessários. Ademais, após 24 dias de exposição química, ocorreu mortalidade e, com 30 dias, houve $100 \%$ de mortalidade em $D$. rerio. Isso alerta para o fato de que a concentração ambiental pode ser letal para organismos mais sensíveis, levando a desequilíbrios populacionais. Khanna e Singh (1983) já relatavam que a glibenclamida provocava em peixes mudanças nas concentrações de metabólitos em tecidos como fígado e rins e no sangue, como hipocolesterolemia, hipoaminoacidemia, queda das concentrações de ácidos graxos livres e fosfolipídios. Adicionalmente, Martínez-Viveros et al. (2018) relataram estresse oxidativo em Cyprinus carpio em diversos tecidos nas concentrações ambientais. Essas alterações metabólicas poderiam ter contribuído para a alta mortalidade observada em $D$. rerio no presente estudo. Ainda, durante a necropsia de $D$. rerio, foi observada congestão nos arcos branquiais e hemorragia na região opercular. MartínezViveros et al. (2018) observaram estresse oxidativo no sangue e nas brânquias de Cyprinus carpio, e Khanna e Singh (1983), mais de 20 anos antes, já relatavam efeitos tóxicos no sangue de peixes, como já descrito acima. Esses 
desequilíbrios somados podem ter contribuído para o aparecimento dos efeitos tóxicos relatados no presente estudo.

Por fim, existem poucos estudos sobre a espécie H. caudovittatus, apesar de sua ampla distribuição na América do Sul. Comparado a $D$. rerio, este se mostrou menos sensível aos testes toxicológicos com os hipoglicemiantes orais (HOs), entretanto $H$. caudovittatus apresenta tamanho um pouco maior que $D$. rerio, o que facilitou o manejo e a coleta de sangue durante os experimentos no presente estudo. Aliado ao baixo custo de aquisição em criadouros comerciais e ao manejo simples em biotério, a espécie se mostrou adequada para testes toxicológicos cujo objetivo seja a avaliação de efeitos de poluentes em animais aquáticos encontrados no Brasil.

\section{CONCLUSÕES}

Pode-se concluir que os hipoglicemiantes orais (HOs) cloridrato de metformina e glibenclamida apresentaram efeitos tóxicos no que se refere a comportamento, glicemia e mortalidade em Hemigrammus caudovittatus e Danio rerio. O D. rerio se mostrou uma espécie mais sensível para estudos com HOs do que o $H$. caudovittatus. Entretanto, recomenda-se o uso desta última espécie em testes toxicológicos. Os HOs foram desenvolvidos para apresentar efeitos fisiológicos em mamíferos, no entanto efeitos tóxicos foram encontrados em peixes. Como existem muitos compostos classificados como HOs que podem chegar até a biota aquática, isto levanta a preocupação sobre seus possíveis efeitos tóxicos e sobre quais métodos serão utilizados para promover sua degradação anterior à chegada ao meio ambiente.

\section{AGRADECIMENTOS}

À Universidade Federal Rural de Pernambuco, por permitir o desenvolvimento desse projeto, e à Facepe (Fundação de Amparo à Ciência e Tecnologia do Estado de Pernambuco), pelo apoio financeiro (APQ-0933-2.08/15).

\section{REFERÊNCIAS}

ALTMANN, J. Observational study of behavior: sampling methods. Behavior, v.48, p.227-267, 1974.

BITTENCOURT, T.Q.M.; SANTOS, A.R.; SILVA, M.C.G. et al. Efeitos tóxicos de compostos de vanádio sobre os parâmetros biológicos de embriões e adultos de zebrafish (Danio rerio). Arq. Bras. Med. Vet. Zootec., v.70, p.1877-1886, 2018.

BOMMER, C.; SAGALOVA, V.; HEESEMANN, E. et al. global economic burden of diabetes in adults: projections from 2015 to 2030. Diabetes Care, v.41, p.963-970, 2018.

CONNAUGHTON, V.P.; BAKER, C.; FONDE, L. et al. alternate immersion in an external glucose solution differentially affects blood sugar values in older versus younger zebrafish adults. Zebrafish, v.13, p.87-94, 2016.

ELO, B.; VILLANO, C.M.; GOVORKO D.; WHITE, L.A. Larval zebrafish as a model for glucose metabolism: expression of phosphoenolpyruvate carboxykinase as a marker for exposure to anti-diabetic compounds. J. Mol. Endocrinol., v.38, p.433-440, 2007.

GLEESON, M.; CONNAUGHTON, V.; ARNESON, L.S. Induction of hyperglycaemia in zebrafish (Danio rerio) leads to morphological changes in the retina. Acta. Diabetol., v.44, p.150-163, 2007.

GRUSH, J.; NOAKES, D.L.; MOCCIA, R.D. The efficacy of clove oil as an anesthetic for the zebrafish. Danio rerio, Zebrafish, v.1, p.46-53, 2004.

KHANNA, N.; SINGH, T. Glibenclamideinduced changes in blood and tissue metabolite levels in fish. Acta Pharmacol. Toxicol., v.52, p.18-21, 1983 .

KUCHARCZYK， D.; TARGONSKA， K.; ZARSKY, D. et al. Reproduction of buenos aires tetra (Hemigrammus caudovittatus) under controlled conditions. Pol. J. Natur. Sci., v.23, p.858-865, 2008. 
LI, S.W.; LIN, A.Y. Increased acute toxicity to fish caused by pharmaceuticals in hospital effluents in a pharmaceutical mixture and after solar irradiation. Chemosphere, v.139, p.190196,2015

LISTA de medicamentos essenciais. [Genebra]: Organização Mundial da Saúde, 2017. Disponível em: <http://www.who.int/medicines/publications/esse ntialmedicines/20th_EML2017_FINAL_amende dAug2017.pdf?ua=1> Acessado em: 27 dez. 2017.

MACLAREN, R.D.; WISNIEWSKI, K.; MACLAREN, C. Environmental concentrations of metformin exposure affect aggressive behavior in the Siamese fighting fish, Betta splendens. Plos One, v.13, n.5, 2018.

MARTÍNEZ-VIVEROS, E.M.G.; ISLASFLORES, H.; DUBRLAN-GARCIA, O. et al. Environmentally relevant concentrations of glibenclamide induce oxidative stress in common carp (Cyprinus carpio). Chemosphere, v.197, p.105-116, 2018.

NIEMUTH, N.J.; JORDAN, R.; CRAGO, J. et al. Metformin exposure at environmentally relevant concentrations causes potential endocrine disruption in adult male fish. Environ. Toxicol. Chem., v.34, p.291-296, 2015.

NIEMUTH, N.J.; KLAPPER, R.D. Low-dose metformin exposure causes changes in expression of endocrine disruption-associated genes. Aquat. Toxicol., v.195, p.33-40, 2018.

OBAIDAT, A.A.; ABABNEH, N.M. Improvement of glibenclamide bioavailability using cyclodextrin inclusion complex dispersed in polyethylene glycol. Jordan. J. Pharm. Sci., v.2, p.119-130, 2009.

PLANCHART, A.; MATTINGLY, C.J.; ALLEN, D. et al. Advancing toxicology research using in vivo high throughput toxicology with small fish models. Altex, v.33, p.435-452, 2016.
PRYOR, R.; CABREIRO, F. Repurposing metformin: an old drug with new tricks in its binding pockets. Biochem. J., v.471, p.307-322, 2015.

ROUBACH, R.; GOMES, L.C.; CHAGAS, E.D. et al. Nutrição e manejo alimentar na piscicultura. Manaus: Embrapa Amazônia Ocidental, 2002, 14 p.

SANTOS, B.D.; SILVA, M.C.G.; SANTOS, T.P. et al. Efeitos de hormônios esteroides de contraceptivos orais combinados sobre os parâmetros comportamentais de Betta splendens (Regan, 1909). Arq. Bras. Med. Vet. Zootec., v.68, p.387-396, 2016.

SCOTT, G.R.; SLOMAN, K.A. The effects of environmental pollutants on complex fish behaviour: integrating behavioural and physiological indicators of toxicity. Aquat. Toxicol., v.68, p.369-392, 2004.

SILVA, J.C.; PACHECO, C.P.; BIZATTO, J. et al. Hipoglicemiantes orais na gestação: metformina versus glibenclamida. Femina, v.37, p.667-670, 2009.

SOARES, P.R.; ANDRADE, A.L.; SANTOS, T.P. et al. Acute and chronic toxicity of the benzoylurea pesticide, lufenuron, in the fish, Colossoma macropomum. Chemosphere, v.16, p.412-421, 2016.

TERNES, T.; BONERZ, M.; SCHMIDT, T. Determination of neutral pharmaceuticals in wastewater and rivers by liquid chromatography-electrospray tandem mass spectrometry. J. Chromatogr. A., v.938, p.175185, 2001.

ZANG, L.; SHIMADA, Y.; NISHIMURA, Y. et al. A Novel, reliable method for repeated blood collection from aquarium fish. Zebrafish, v.10, p.425-432, 2013.

ZIELINSK, F.; SAGER, N.; CHIU, Y. Review of environmental assessment for metaglip tablets (Glipizide and Metformin $\mathrm{HCl}$ ). Center Drug Eval. Res., p.2-8, 2002. 